\title{
Osimertinib for EGFR-mutant lung cancer with central nervous system metastases: a meta-analysis and systematic review
}

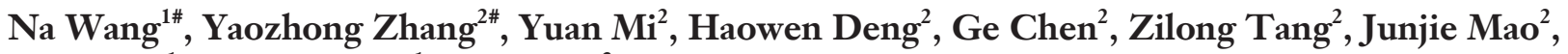 \\ Saijin Cui ${ }^{1}$, Yaling Zhang ${ }^{1}$, Lei Wang ${ }^{2}$ \\ ${ }^{1}$ Cancer Institute, ${ }^{2}$ Department of Thoracic Surgery, The Fourth Hospital of Hebei Medical University, Shijiazhuang, China \\ Contributions: (I) Conception and design: N Wang, L Wang; (II) Administrative support: N Wang, L Wang; (III) Provision of study materials or \\ patients: H Deng; (IV) Collection and assembly of data: Z Tang, J Mao; (V) Data analysis and interpretation: Y Zhang; (VI) Manuscript writing: All \\ authors; (VII) Final approval of manuscript: All authors. \\ "These authors contributed equally to this work. \\ Correspondence to: Lei Wang. Department of Thoracic Surgery, The Fourth Hospital of Hebei Medical University, Shijiazhuang, China. \\ Email: yuankundu@163.com.
}

Background: Osimertinib, a third-generation tyrosine kinase inhibitor (TKI), is the only Food and Drug Administration-approved third-generation epidermal growth factor receptor (EGFR)TKI. Osimertinib is a cancer medicine that interferes with the growth and spread of cancer cells in the body. Osimertinib is used to treat a certain type of non-small cell lung cancer. We review some of the main challenges in targeting EGFR, including lack of central nervous system penetration with most tyrosine kinase inhibitors, activity of osimertinib penetrating blood-brain barrier and the efficacy of osimertinib.

Methods: Guided by the preferred reporting items for systematic reviews and meta-analyses (PRISMA) statement, we conducted a systematic literature search in the databases PubMed, EMBASE, ISI Web of Science database on January 30, 2020, searching for studies investigating the osimertinib efficacy on patients with CNS metastases in EGFR-mutant non-small cell lung cancer (NSCLC). And Newcastle-Ottawa Scale (NOS) was used to assess the certainty in the evidence.

Results: The pooled results showed that the overall response rate (ORR) and disease control rate (DCR) were $70 \%$ and $92 \%$, respectively, in patients with T790M mutations. The efficacy of osimertinib was confirmed by the median progression free survival (PFS). In untreated advanced EGFR-mutated NSCLC with CNS metastases patients, the pooled ORR and DCR of osimertinib were $71 \%$ and $93 \%$, respectively. And the combined median PFS, achieved by osimertinib, was 12.21 months. Above data proved that osimertinib has well activity in disease control, especially in first line.

Conclusions: This meta-analysis confirmed that in treatment-naive advanced NSCLC CNS metastases harboring EGFR-TKI-sensitizing mutations, Osimertinib showed impressive antitumor activity.

Keywords: Osimertinib; EGFR-mutant lung cancer; central nervous system metastases; meta-analysis

Submitted Mar 10, 2020. Accepted for publication Jul 28, 2020.

doi: 10.21037/apm-20-605

View this article at: http://dx.doi.org/10.21037/apm-20-605

\section{Introduction}

Brain metastasis of lung cancer is common in a lot of patients with non-small cell lung cancer (NSCLC) and may affect negatively survival and quality of life (1). Epidermal growth factor receptor (EGFR), widely distributed in human epidermal cells and stromal cells, is an important membrane surface receptor with tyrosine-kinase activity (2). EGFR exon 19 deletions and point mutations in exon 21 can be found in a lot of NSCLC patients (3). Actually, a variety of EGFR-sensitizing mutations were harbored in about $10-40 \%$ of NSCLC patients. Therefore, these vital 
therapeutic mutations are considered important for the advanced NSCLC.

The question is that it can't be said to be satisfactory that the efficiency of first- and second-generation epidermal growth factor receptor tyrosine kinase inhibitors (EGFRTKIs) in central nervous system (CNS) metastasis (4). Preclinical models and clinical studies had shown that these agents have limited ability to cross the blood-brain barrier, allowing the CNS to emerge as a sanctuary site for metastatic spread. Patients treated with first- or secondgeneration EGFR-TKIs were often with impressive initial response, but exhibit progression after $10-14$ months $(5,6)$. Osimertinib $(7,8)$ is a third-generation tyrosine kinase inhibitor (TKI), initially approved for epidermal growth factor receptor (EGFR) mutant non-small cell lung cancer (NSCLC) with T790M acquired resistance.

A meta-analysis, about osimertinib in treating EGFRmutated advanced NSCLC, showed that subgroup analysis in the AURA3 study suggested that osimertinib had a higher CNS response rate and prolonged CNS PFS compared to those of chemotherapy (9). However, osimertinib compared to first- and second-generation EGFR-TKIs. Data supporting the use of osimertinib in brain metastases are limited, although it had established better CNS activity in some preclinical models and clinical studies. There is no aggregate analysis regarding CNS metastases performed. Therefore, our meta-analysis will be the first systematic review to synthesize the results of published literature, efficiency comparison between osimertinib and other drug therapy, to provide more objective data for the optimal clinical use of osimertinib in CNS metastasis of NSCLC. We present the following article in accordance with the PRISMA Reporting Checklist (available at http://dx.doi. org/10.21037/apm-20-605).

\section{Methods}

\section{Search strategy}

We have registered at International Prospective Register of Systematic Reviews (CRD 42019125600). Some database, including PubMed, EMBASE, ISI Web of Science database and Cochrane Library, were searched for eligible studies. The last retrieval was performed on January 30, 2020. The following search terms were used for the literature search: ("osimertinib" OR "mereletinib" OR “AZD9291" OR "Tagrisso") AND ("Non-Small Cell Lung Cancer" OR "Non-Small Cell Lung Carcinoma" OR "Non-Small Cell
Lung Carcinoma" OR "Non-Small-Cell Lung Carcinoma" OR "Non-small Cell Lung Cancer" OR "Non-Small-Cell Lung Carcinomas" OR "Non-Small Cell Lung Carcinoma" OR "NSCLC") AND ("Brain" OR "CNS" OR “central nervous system"). The titles and abstracts of the primary studies identified in the electronic search were retrieved by two authors independently.

\section{Inclusion and exclusion criteria}

The inclusion criteria are as follows: (I) the studies were trials performed about osimertinib in NSCLC patients with CNS metastasis confirmed by magnetic resonance imaging (MRI) or computed tomography (CT); (II) we can obtain response rate or other relevant data from the identified study to calculate; (III) written in English; and we only use the data of the most recent trial were used when the reported data were from the same patients. We have deleted all reviews, case reports, animal or duplicate publications.

\section{Data extraction and quality assessment}

Two authors independently extracted data and evaluated quality of the literature. We extracted the following information: first name of the author, year of publication, region, trial design, sub-category, EGFR mutant (\%), age (years), sample size, dosage and length of osimertinib, tumor response, data of PFS. The NOS standard (10) was used as a research quality assessment standard. Low quality means that studies with a score no more than 4. The two authors resolved the problem, such a difference in opinion on a document, through mutual discussion and requested help from a third author if necessary.

\section{Statistical analysis}

All eligible studies were included. The heterogeneity between studies was evaluated with $\mathrm{Q}$ and $\mathrm{I}^{2}$ statistics (11). Studies with an $\mathrm{I}^{2}$ statistics of $0 \%, 25 \%$ and $50 \%$ represented no, low and moderate, respectively. High heterogeneity meant that $\mathrm{I}^{2}$ was more than $75 \%$. According to the results of inter-study heterogeneity appraisal using $\mathrm{Q}$ and $\mathrm{I}^{2}$ statistics, Fixed-effects model was preferred to random-effects model when there was no statistically moderate heterogeneity $\left(\mathrm{I}^{2}>50 \%\right)$ between studies, and vice versa when there was high heterogeneity (12). We performed the integrated analysis to calculate the $95 \%$ confidence interval (CI), representing effect size. According 


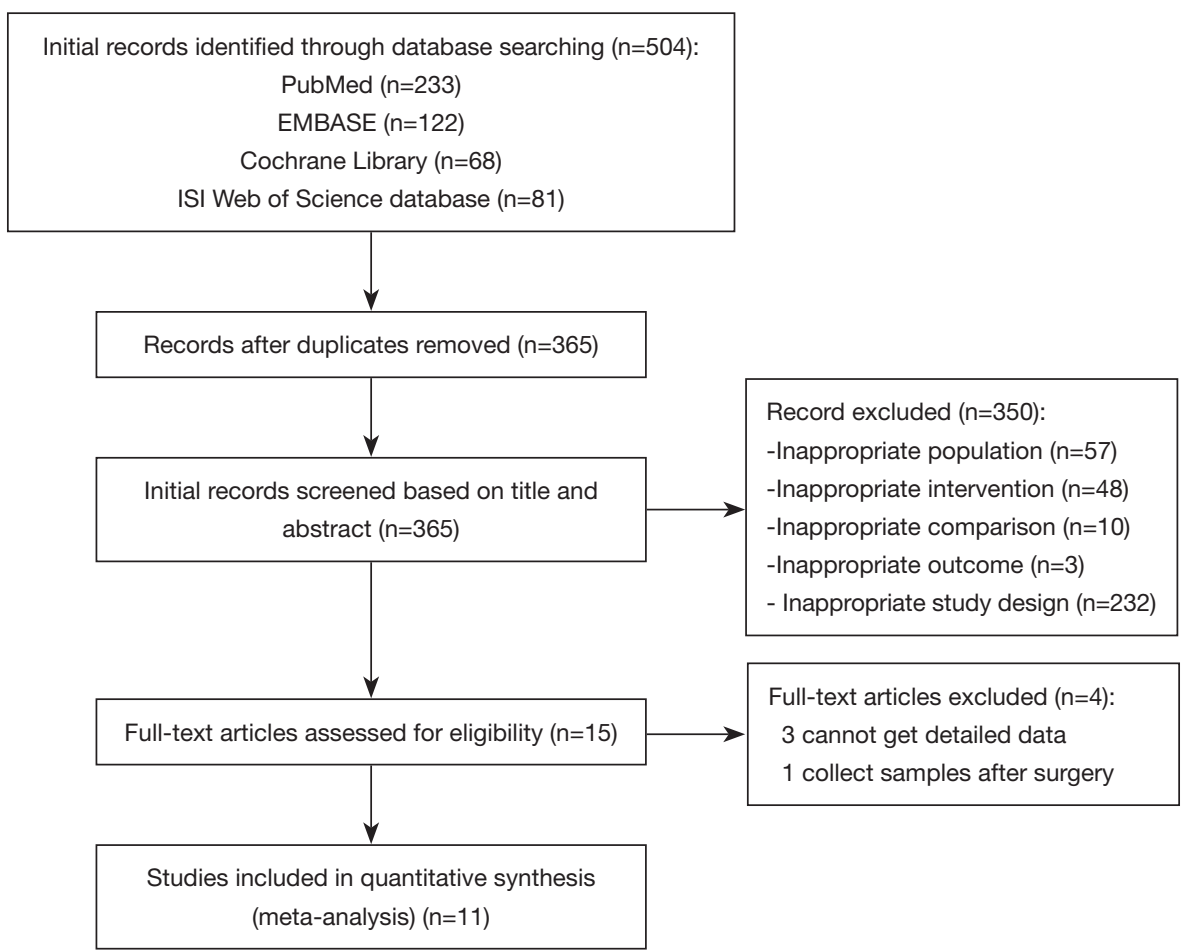

Figure 1 Search strategy and identification of studies. From: Moher D, Liberati A, Tetzlaff J, Altman DG, The PRISMA Group. Preferred Reporting Items for Systematic Reviews and Meta-Analyses: The PRISMA Statement. PLoS Med 2009;6:e1000097. doi:10.1371/journal. pmed1000097. For more information, visit www.prisma-statement.org.

to treatment lines, the analysis parameter included all data of response or survival. We also conducted subgroup analyses of objective response rate (ORR) and disease control rate (DCR) to the dose of osimertinib. In addition, we have performed sensitivity analysis by excluding the studies with the lowest-quality score. If necessary, the heterogeneity was also explored by subgroup. The potential publication bias was further validated by the Egger's and Begg's test (13). All statistical analyses were two sides. A P value less than 0.05 was considered statistically significant. This meta-analysis was conducted by the STATA version 14.0 (Stata Corporation, College Station, TX).

\section{Results}

\section{Search results}

The PRISMA flow chart of this meta-analysis is shown in Figure 1. Duplicates and irrelevant studies and those without sufficient data were removed from a total of 504 publications. All investigators finally agreed to include 11 eligible (14-24) studies with 842 patients in our meta- analysis (Table 1). There are four RCTs, three singlearm trials, two retrospective studies and two real-world treatment studies. The eligible studies were published from 2017 to 2019, and average age (median or mean) in the included studies was ranged from 24.9 to 65 years. The sample size was ranged from 24 to 211 . All studies included osimertinib $80 \mathrm{mg}$ dose.

\section{ORR and DCR}

ORR of osimertinib was obtained from eleven eligible studies. The overall ORR of osimertinib, in analysis, was $70 \%$ (95\% CI: $68-71 \%)$. According to the line of treatment, we conducted further analysis on the ORR. Five studies provided data on first-line treatment and other six provided data on second-line treatment or beyond. In analysis, the pooled ORR of patients were $71 \%$ (95\% CI: 69-73\%) with moderate heterogeneity $\left(\mathrm{I}^{2}=0 \%, \mathrm{P}=0.65\right)$, and $65 \%$ (95\% CI: 62-68\%) with no heterogeneity $\left(\mathrm{I}^{2}=0 \%, \mathrm{P}=0.63\right.$; Figure 2), in first-line and $\geq$ second-line treatment, respectively. All selected studies provided data on DCR, 


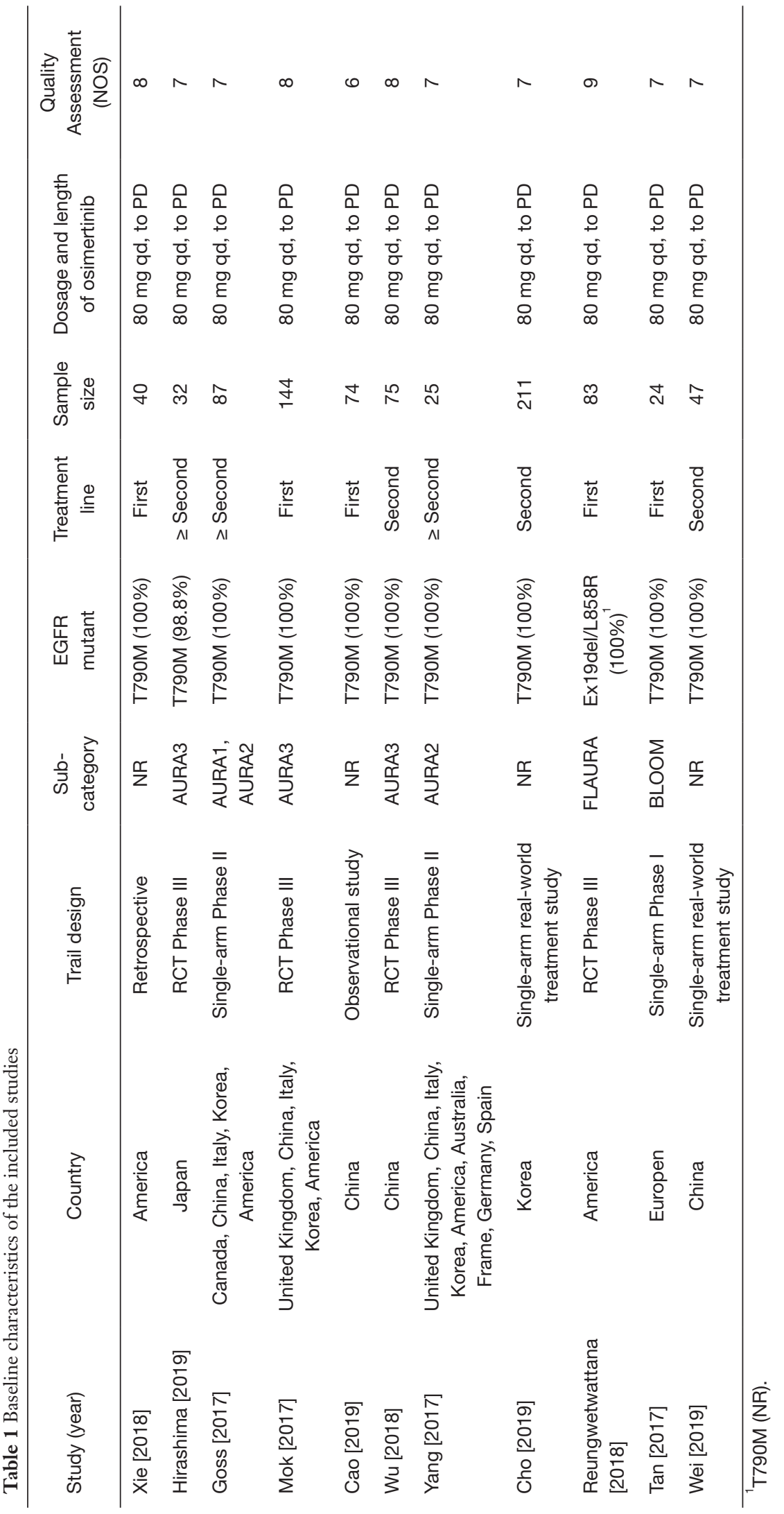




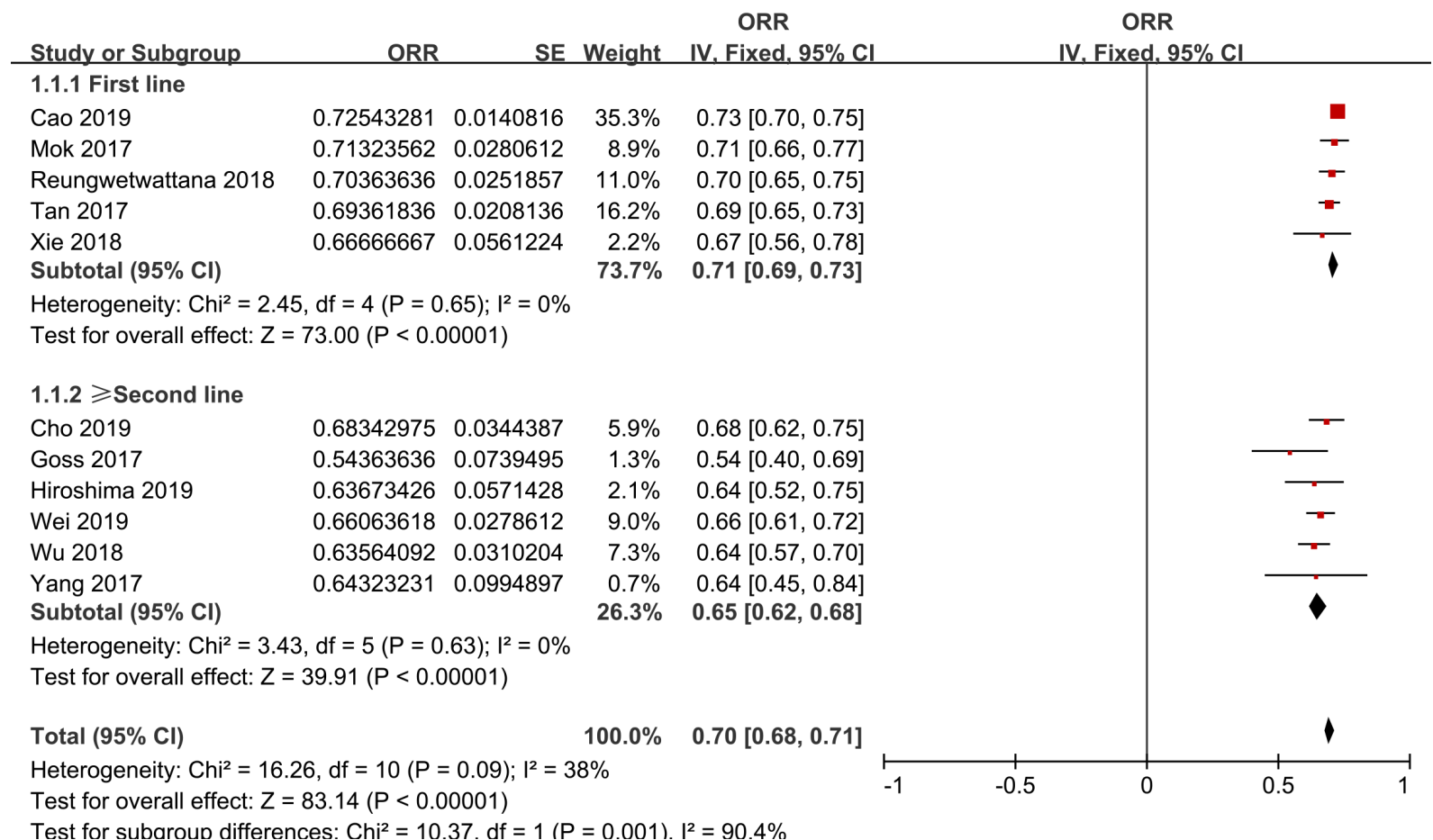

Figure 2 Overall response rate (ORR) of NSCLC CNS metastasis treated with osimertinib.

and the overall DCR was $92 \%$ (95\% CI: 91-94\%). The combined DCR was 93\% (95\% CI: $90-95 \%),\left(\mathrm{I}^{2}=0 \%\right.$, $\mathrm{P}=0.86)$, and $92 \%$ (95\% CI: 89-94\%), $\left(\mathrm{I}^{2}=0 \%, \mathrm{P}=0.88\right.$; Figure 3) in first-line treatment group and second-line treatment or beyond, respectively.

The data on complete response (CR), partial response (PR), and stable disease (SD) were obtained from five studies $(14,16,18,19,22)$. CR meant that all lesions disappeared, no new lesions appeared and tumor markers were normal, for at least 4 weeks. This analysis suggested that the pooled CR values of the first-line group and the second-line or beyond group were $3 \%$ (95\% CI: $1-4 \%)\left(\mathrm{I}^{2}=0 \%, \mathrm{P}=0.74\right)$ and $2 \%$ (95\% CI: $1-5 \%)\left(\mathrm{I}^{2}=79 \%, \mathrm{P}=0.003\right)$, with overall CR $2 \%$ (95\% CI: $1-4 \%$ ). PR was defined as the reduction of the target focus maximum diameter $\geq 30 \%$. The pooled PR was $62 \%$ (95\% CI: $39-84 \%$ ). Subgroup analysis showed that the pooled PR of the first-line group was $77 \%$ (95\% CI: $72-81 \%)\left(\mathrm{I}^{2}=0 \%, \mathrm{P}=0.51\right)$. However, $\mathrm{PR}$ of the second-line or beyond group significantly reduced, only $55 \%$ (95\% CI: $27-84 \%)\left(\mathrm{I}^{2}=99 \%, \mathrm{P}<0.00001\right)$. The pooled $\mathrm{SD}$ was $15 \%$ (95\% CI: 9-21\%). Our research showed that the pooled SD of the first-line group was $17 \%$ (95\% CI: $13-21 \%)$, and no heterogeneity was found $\left(\mathrm{I}^{2}=0 \%, \mathrm{P}=0.58\right)$. on the contrary, the pooled SD of the second-line or beyond group was
14\% (95\% CI: 5-22\%), with high heterogeneity $\left(\mathrm{I}^{2}=94 \%\right.$, $\mathrm{P}<0.00001)$.

\section{Progression-free survival}

The pooled median PFS was 10.98 months (95\% CI: 9.4312.53 months; Figure 4A). Through subgroup analysis, the pooled median PFS of untreated EGFR CNS metastasis patients was 12.21 months (95\% CI: 9.20-15.23 months) $\left(\mathrm{I}^{2}=88 \%, \mathrm{P}<0.00001\right)$. While, the pooled median PFS of second line or beyond was only 10.10 months (95\% CI: $8.87-11.33$ months) ( $\left.\mathrm{I}^{2}=23 \%, \mathrm{P}=0.26\right)$.

Based on the data from eligible studies, we analyzed PFS-6 and PFS-12 separately. The pooled PFS-6, based on four studies, was 64\% (95\% CI: 48-80\%). The pooled PFS-6 was $74 \%$ (95\% CI: 66-81\%), $\left(\mathrm{I}^{2}=69 \%, \mathrm{P}=0.04\right)$ and $55 \%$ (95\% CI: $37-73 \%),\left(\mathrm{I}^{2}=99 \%, \mathrm{P}<0.00001\right.$; Figure $\left.4 B\right)$, in the first-line group and second-line or beyond group. Meanwhile, the combined PFS-12 was 39\% (95\% CI: $22-57 \%)$. Subgroup analysis indicated that the pooled PFS12 was $52 \%$ (95\% CI: $43-61 \%),\left(\mathrm{I}^{2}=77 \%, \mathrm{P}=0.01\right)$ and $30 \%$ (95\% CI: $12-48 \%),\left(\mathrm{I}^{2}=99 \%, \mathrm{P}<0.00001\right.$; Figure 4 C), in the first-line group and the second-line or beyond group, respectively. However, the results were unstable during the 


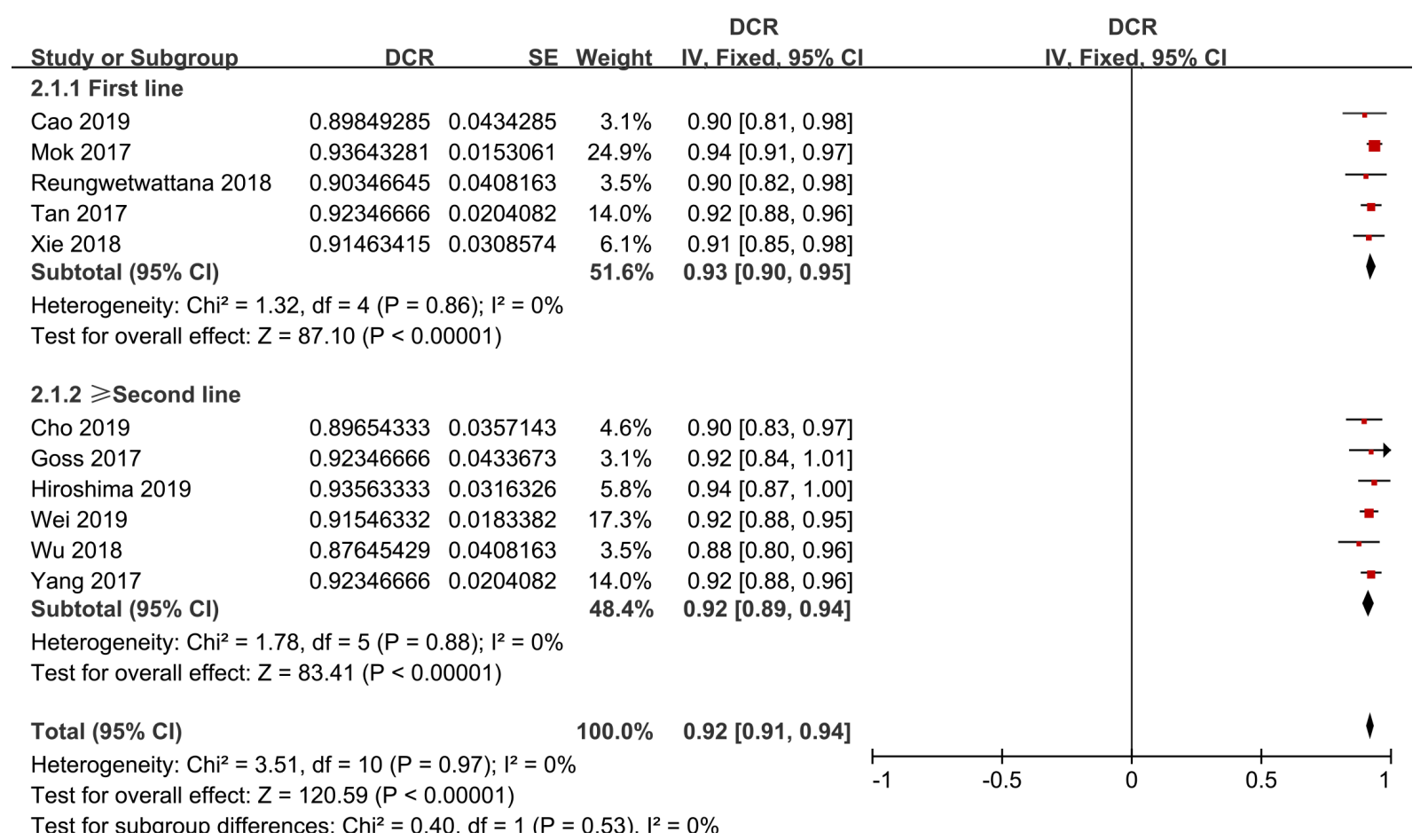

Figure 3 Disease control rate (DCR) of NSCLC CNS metastasis treated with osimertinib.

pooled analysis of PFS-12 in second-line treatment group. When two articles by Wu et al. (19) and Yang et al. (20) were removed, the research outcome changed, $43 \%$ (95\% CI: $38-49 \%)$ with moderate heterogeneity $\left(\mathrm{I}^{2}=63 \%\right.$, $\mathrm{P}=0.07)$. This may be explained by the fact that there are no patients after radiotherapy in three other articles $(15,16,21)$, whereas both Wu et al. (19) and Yang et al. (20) included patients who received brain radiotherapy within 6 months.

The publication bias in this meta-analysis was indicated by Egger's test and Begg's test. The results are shown in Table 2. Both two tests confirmed that no significantly different results emerged. The conclusions were not changed after adjustment for publication bias by using the trim and fill method (25).

\section{Discussion}

Eleven research literatures, totally 842 patients, were involved by our study included to evaluate the efficacy and safety of osimertinib in treating CNS metastases in EGFRmutant non small cell lung cancer (NSCLC) patients. The high ORR and DCR confirmed the efficacy of osimertinib in treating EGFR-mutated NSCLC with CNS metastases patients. Special attention should be paid to the higher
ORR and DCR, $71 \%$ and $93 \%$, in first-line therapy. And the combined median PFS of osimertinib for untreated patients was 12.21 months. Above data suggested that osimertinib provided good disease control on advanced EGFR-mutated NSCLC with CNS metastases, especially for untreated patients.

EGFR TKIs inhibit the proliferation of tumor cells via binding to EGFR specifically and show favorable therapeutic effects on advanced EGFR-mutated NSCLC. Tumors sensitive to EGFR TKIs $(26,27)$ harbored activating mutations of EGFR (exon 19 deletion or L858R point mutation in exon 21). As shown above, osimertinib is of extraordinary efficacy both in ORR, DCR and PFS, and is better than afatinib with only median PFS: 8.2 months (5). Drug resistance largely limited the effectiveness of EGFR TKIs. Although both first-generation (gefitinib and erlotinib) and second-generation (afatinib) EGFR TKIs have demonstrable clinical efficacy in patients with EGFRactivating mutations, producing improvements in response rates (RRs), time to progression, and overall survival (OS), most patients develop acquired resistance following 10-14 months of treatment. The acquisition of a secondary T790M mutation in exon 20 of the EGFR gene caused many patients developed resistance to EGFR TKIs (28). 


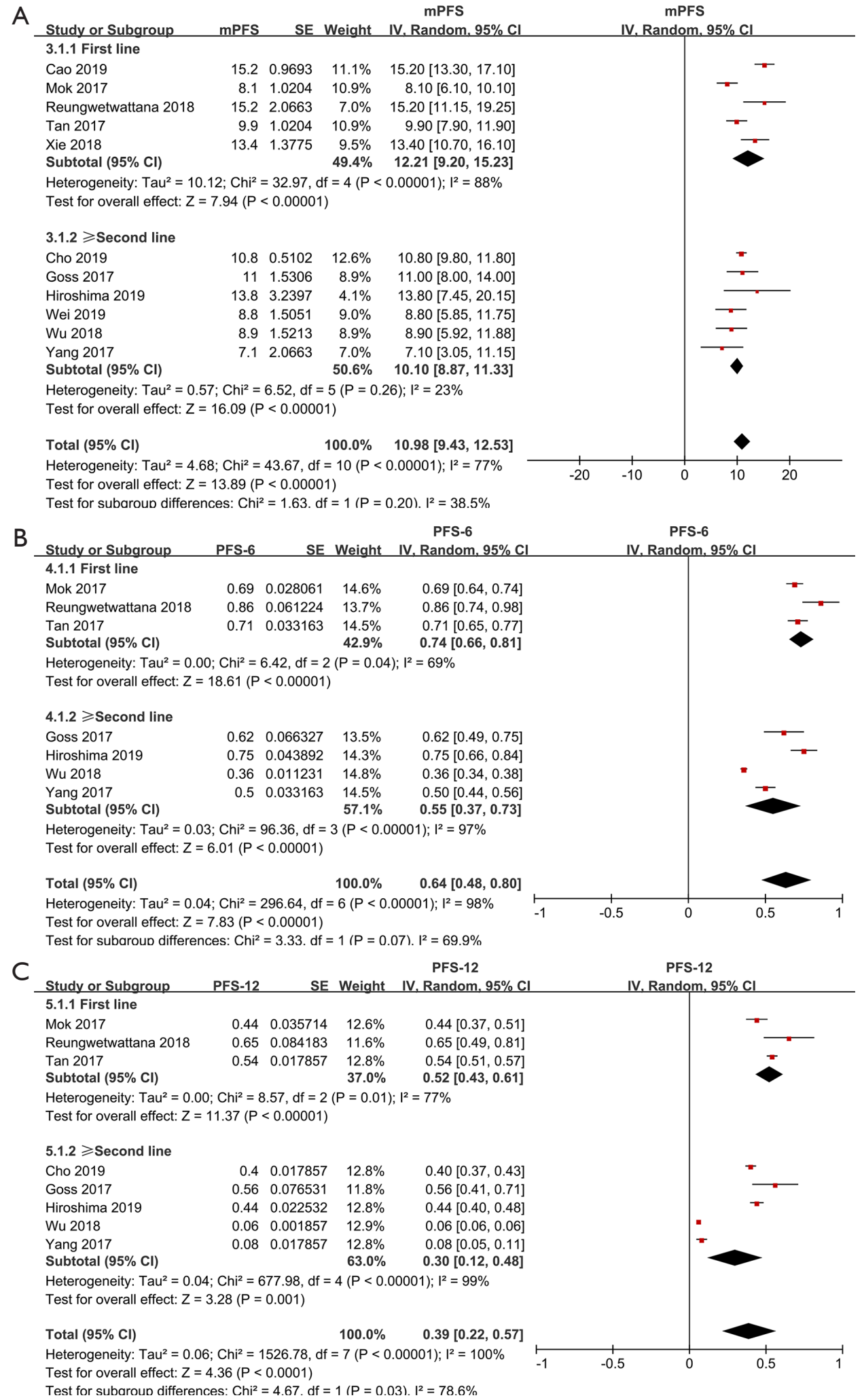

Figure 4 Median progression-free survival (PFS), 6-month progression-free survival (PFS-6), and 12-month progression free survival (PFS12) of NSCLC CNS metastasis treated with osimertinib. 
Table 2 Result of publication bias

\begin{tabular}{lcc}
\hline Item & Egger's test & Begg's test \\
\hline ORR & 0.511 & 0.764 \\
DCR & 0.220 & 0.448 \\
mPFS & 0.403 & 0.322 \\
PFS-6 & 0.864 & 0.760 \\
PFS-12 & 0.033 & 0.732 \\
\hline
\end{tabular}

We found that T790M mutation was observed in more than $50 \%$ of patients who developed resistance to an EGFR TKIs. However, the T790M status of patients' CNS disease was not assessed. Published data suggest that T790M may occur less frequently in CNS metastases than in the primary tumour; therefore, some patients in our study may have had T790M-negative CNS metastases. In such patients, osimertinib would not be acting against the T790M resistance mutation. Neurological deficits and devastating clinical reality will be caused by the metastasis of cancer to the CNS, with an estimated survival time of less than one year.

As an orally taken third-generation EGFR-TKIs, Osimertinib can form an irreversible covalent bond with T790M or other EGFR mutations, via the cysteine-797 residue. Osimertinib also was the only one EGFR-TKIs, by Food and Drug Administration, approved for the treatment of patients with metastatic T790M mutation positive NSCLC, and displayed impressive activity in blood brain barrier transmigration. Yi et al.'s study suggested that osimertinib had impressive response rates in treatmentnaive advanced NSCLC harboring EGFR-TKI sensitizing mutations with tolerable AEs (9), and the analysis by dose indicated that $80 \mathrm{mg}$ of osimertinib once daily had higher ORR and DCR than the other doses. What's more, osimertinib demonstrated consistently superior CNS efficacy compared with platinum-pemetrexed $(17,19)$. For no treatment EGFR-mutated advanced NSCLC CNS patients, efficacy of osimertinib is also better $(22,23)$. In trial, the frequency of events of CNS progression at the time of this analysis was lower in the osimertinib group than in the standard EGFR TKIs group (23). Compared with patients who had not receive prior brain radiotherapy or radiotherapy $\geq 6$ months before osimertinib, the observed ORR was lower in patients who received radiotherapy $\geq 6$ months before osimertinib $(16,19,20)$. CNS efficacy of osimertinib was observed regardless of prior radiotherapy status. Some case reports had demonstrated that the clinical responses achieved on osimertinib may obviate the need for whole-brain radiation in some patients (29-31). Receiving radiation prior to starting osimertinib for patients with progressing brain metastases did not prolong PFS or OS (32). To minimize the risks of radiation-related toxicity, delaying radiation could be considered for some patients with EGFR-mutant NSCLC with brain metastases who initially respond to osimertinib (14).

Due to the blood-brain barrier, the efficacy of the standard cytotoxic chemotherapy (mainly platinum doublets or first- and second-generation EGFR-TKIs) for NSCLC is limited in the treatment of brain metastases (33). Osimertinib was effective in improving overall survival, post progression survival and brain metastasis-free survival in meta-analysis (34).

We acknowledge several limitations to this. First, to a certain extent, high heterogeneity limited test efficiency. Although several subgroup analyses were performed, significant heterogeneity was generally observed. Given the differences of the studies, there is a greater risk of potential selection and reporting bias. Second, limited number of studies and small sample size are limitations of our study, which might cause a result in invalid statistical analyses in those groups. Third, several sources of bias, such as inherent limitations, unmeasured confounding, and the typical bias in observational studies, may influence the observed results.

\section{Conclusions}

The results of our study indicate that most patients with CNS metastases would respond to osimertinib treatment in NSCLC harboring T790M mutations. Moreover, osimertinib has impressive antitumor activity in treatmentnaive advanced NSCLC CNS metastases harboring EGFRTKI-sensitizing mutations. However, more randomized clinical trials and additional fundamental researches are still needed to further clarify the beneficial population of different therapy and its possible mechanism, so as to better guide clinical treatment.

\section{Acknowledgments}

We sincerely thank all the reviewers and editors for their supportive suggestions.

Funding: This study was supported by the Key Medical Research Fund of Hebei Provincial Health Planning Commission (20160177 and 20180528). 


\section{Footnote}

Reporting Checklist: The authors have completed the PRISMA Reporting Checklist. Available at http://dx.doi. org/10.21037/apm-20-605

Conflicts of Interest: All authors have completed the ICMJE uniform disclosure form (available at http://dx.doi. org/10.21037/apm-20-605). The authors have no conflicts of interest to declare.

Ethical Statement: The authors are accountable for all aspects of the work in ensuring that questions related to the accuracy or integrity of any part of the work are appropriately investigated and resolved.

Open Access Statement: This is an Open Access article distributed in accordance with the Creative Commons Attribution-NonCommercial-NoDerivs 4.0 International License (CC BY-NC-ND 4.0), which permits the noncommercial replication and distribution of the article with the strict proviso that no changes or edits are made and the original work is properly cited (including links to both the formal publication through the relevant DOI and the license). See: https://creativecommons.org/licenses/by-nc-nd/4.0/.

\section{References}

1. Shaikh SS, Kumthekar PU, Mohindra NA. A review of eligibility for patients with central nervous system (CNS) metastases from non-small cell lung cancer (NSCLC) in immunotherapy clinical trials. J Neurooncol 2019;144:235-7.

2. Liu X, Wang $P$, Zhang C, et al. Epidermal growth factor receptor (EGFR): A rising star in the era of precision medicine of lung cancer. Oncotarget 2017;8:50209-20.

3. Jiang H, Zhu M, Li Y, et al. Association between EGFR exon 19 or exon 21 mutations and survival rates after firstline EGFR-TKI treatment in patients with non-small cell lung cancer. Mol Clin Oncol 2019;11:301-8.

4. Kelly WJ, Shah NJ, Subramaniam DS. Management of Brain Metastases in Epidermal Growth Factor Receptor Mutant Non-Small-Cell Lung Cancer. Front Oncol 2018;8:208.

5. Yang JC, Wu YL, Schuler M, et al. Afatinib versus cisplatin-based chemotherapy for EGFR mutation-positive lung adenocarcinoma (LUX-Lung 3 and LUX-Lung 6): analysis of overall survival data from two randomised, phase 3 trials. Lancet Oncol 2015;16:141-51.

6. Park K, Tan EH, O'Byrne K, et al. Afatinib versus gefitinib as first-line treatment of patients with EGFR mutation-positive non-small-cell lung cancer (LUX-Lung 7): a phase $2 \mathrm{~B}$, open-label, randomised controlled trial. Lancet Oncol 2016;17:577-89.

7. Lamb YN, Scott LJ. Osimertinib: A Review in T790MPositive Advanced Non-Small Cell Lung Cancer. Target Oncol 2017;12:555-62.

8. Soria JC, Ohe Y, Vansteenkiste J, et al. Osimertinib in Untreated EGFR-Mutated Advanced Non-Small-Cell Lung Cancer. N Engl J Med 2018;378:113-25.

9. Yi L, Fan J, Qian R, et al. Efficacy and safety of osimertinib in treating EGFR-mutated advanced NSCLC: A meta-analysis. Int J Cancer 2019;145:284-94.

10. Stang A. Critical evaluation of the Newcastle-Ottawa scale for the assessment of the quality of nonrandomized studies in meta-analyses. Eur J Epidemiol 2010;25:603-5.

11. Takkouche B, Khudyakov P, Costa-Bouzas J, et al. Confidence intervals for heterogeneity measures in metaanalysis. Am J Epidemiol 2013;178:993-1004.

12. Schmidt FL. History and development of the SchmidtHunter meta-analysis methods. Res Synth Methods 2015;6:232-9.

13. Lin L, Chu H. Quantifying publication bias in metaanalysis. Biometrics 2018;74:785-94.

14. Xie L, Nagpal S, Wakelee HA, et al. Osimertinib for EGFR-Mutant Lung Cancer with Brain Metastases: Results from a Single-Center Retrospective Study. Oncologist 2019;24:836-43.

15. Hirashima T, Satouchi M, Hida T. Osimertinib for Japanese patients with T790M-positive advanced nonsmall-cell lung cancer: A pooled subgroup analysis. Cancer Sci 2019;110:2884-93.

16. Goss G, Tsai CM, Shepherd FA, et al. CNS response to osimertinib in patients with T790M-positive advanced NSCLC: pooled data from two phase II trials. Ann Oncol 2018;29:687-93.

17. Mok TS, Wu YL, Ahn MJ, et al. Osimertinib or PlatinumPemetrexed in EGFR T790M-Positive Lung Cancer. N Engl J Med 2017;376:629-40.

18. Cao Y, Qiu X, Xiao G, et al. Effectiveness and safety of osimertinib in patients with metastatic EGFR T790Mpositive NSCLC: An observational real-world study. PLoS One 2019;14:e0221575.

19. Wu YL, Ahn MJ, Garassino MC, et al. CNS Efficacy of Osimertinib in Patients With T790M-Positive Advanced Non-Small-Cell Lung Cancer: Data From a Randomized 
Phase III Trial (AURA3). J Clin Oncol 2018;36:2702-9.

20. Yang JC, Ahn MJ, Kim DW, et al. Osimertinib in Pretreated T790M-Positive Advanced Non-SmallCell Lung Cancer: AURA Study Phase II Extension Component. J Clin Oncol 2017;35:1288-96.

21. Cho BC, Kim DW, Park K, et al. Real-world use of osimertinib in non-small cell lung cancer: ASTRIS study Korean subgroup analysis. Curr Med Res Opin 2020;36:477-82.

22. Reungwetwattana T, Nakagawa K, Cho BC, et al. CNS Response to Osimertinib Versus Standard Epidermal Growth Factor Receptor Tyrosine Kinase Inhibitors in Patients With Untreated EGFR-Mutated Advanced Non-Small-Cell Lung Cancer. J Clin Oncol 2018:Jco2018783118. [Epub ahead of print].

23. Tan CS, Cho BC, Soo RA. Treatment options for EGFR mutant NSCLC with CNS involvement-Can patients BLOOM with the use of next generation EGFR TKIs? Lung Cancer 2017;108:29-37.

24. Wei B, Zhao C, Li J, et al. Combined plasma and tissue genotyping of EGFR T790M benefits NSCLC patients: a real-world clinical example. Mol Oncol 2019;13:1226-34.

25. Mavridis D, Salanti G. How to assess publication bias: funnel plot, trim-and-fill method and selection models. Evid Based Ment Health 2014;17:30.

26. Oxnard GR, Arcila ME, Chmielecki J, et al. New strategies in overcoming acquired resistance to epidermal growth factor receptor tyrosine kinase inhibitors in lung cancer. Clin Cancer Res 2011;17:5530-7.

27. Russo A, Franchina T, Ricciardi GRR, et al. Third generation EGFR TKIs in EGFR-mutated NSCLC: Where are we now and where are we going. Crit Rev Oncol Hematol 2017;117:38-47.

Cite this article as: Wang N, Zhang Y, Mi Y, Deng H, Chen G, Tang Z, Mao J, Cui S, Zhang Y, Wang L. Osimertinib for EGFR-mutant lung cancer with central nervous system metastases: a meta-analysis and systematic review. Ann Palliat Med 2020;9(5):3038-3047. doi: 10.21037/apm-20-605
28. Yu HA, Arcila ME, Rekhtman N, et al. Analysis of tumor specimens at the time of acquired resistance to EGFR-TKI therapy in 155 patients with EGFR-mutant lung cancers. Clin Cancer Res 2013;19:2240-7.

29. Reichegger H, Jochum W, Forbs D, et al. Rapid Intracranial Response to Osimertinib in a Patient with Epidermal Growth Factor Receptor T790MPositive Adenocarcinoma of the Lung. Oncol Res Treat 2016;39:461-3.

30. Ricciuti B, Chiari R, Chiarini P, et al. Osimertinib (AZD9291) and CNS Response in Two RadiotherapyNaive Patients with EGFR-Mutant and T790M-Positive Advanced Non-Small Cell Lung Cancer. Clin Drug Investig 2016;36:683-6.

31. Senoo S, Ohashi K, Nishii K, et al. Osimertinib Depletes EGFR T790M in the Spinal Fluid of Patients with Carcinomatous Meningitis of Lung Adenocarcinoma Harboring De Novo EGFR T790M. J Thorac Oncol 2018;13:e140-2.

32. Yousefi M, Bahrami T, Salmaninejad A, et al. Lung cancer-associated brain metastasis: Molecular mechanisms and therapeutic options. Cell Oncol (Dordr) 2017;40:419-41.

33. Ballard P, Yates JWT, Yang Z, et al. Preclinical Comparison of Osimertinib with Other EGFR-TKIs in EGFR-Mutant NSCLC Brain Metastases Models, and Early Evidence of Clinical Brain Metastases Activity. Clin Cancer Res 2016;22:5130-40.

34. Joo JW, Hong MH, Shim HS. Clinical characteristics of T790M-positive lung adenocarcinoma after resistance to epidermal growth factor receptor-tyrosine kinase inhibitors with an emphasis on brain metastasis and survival. Lung Cancer 2018;121:12-7. 